\title{
HAMILTON-JACOBI EQUATIONS WITH SINGULAR BOUNDARY CONDITIONS ON A FREE BOUNDARY AND APPLICATIONS TO DIFFERENTIAL GAMES
}

\author{
MARTINO BARDI AND PIERPAOLO SORAVIA
}

\begin{abstract}
A class of Hamilton-Jacobi equations arising in generalized timeoptimal control problems and differential games is considered. The natural global boundary value problem for these equations has a singular boundary condition on a free boundary. The uniqueness of the continuous solution and of the free boundary is proved in the framework of viscosity solutions. A local uniqueness theorem is also given, as well as some existence results and several applications to control and game theory. In particular a relaxation theorem (weak form of the bang-bang principle) is proved for a class of nonlinear differential games.
\end{abstract}

\section{INTRODUCTION}

In this paper we study viscosity solutions of Hamilton-Jacobi $(\mathrm{HJ})$ equations of the form

$$
H(x, D U)=0,
$$

for Hamiltonians admitting the representation

$$
H(x, p):=\min _{b \in B} \max _{a \in A}\{-f(x, a, b) \cdot p-h(x, a, b)\} \quad \text { for all } x, p \in \mathbf{R}^{N}
$$

with $A, B$ compact, $f, h$ sufficiently smooth, and $h$ satisfying the condition

$$
h(x, a, b) \geq h_{0}>0 \text { for all } x \in \mathbf{R}^{N}, a \in A, b \in B .
$$

We prove two types of uniqueness results which seem to be characteristic of this particular class of $\mathrm{HJ}$ equations. The first problem we consider is

$$
\begin{cases}H(x, D U)=0 & \text { in } \Omega \backslash \mathscr{T}, \\ U=g & \text { on } \partial \mathscr{T}, \\ U(x) \rightarrow+\infty & \text { as } x \rightarrow x_{0} \in \partial \Omega,\end{cases}
$$

where $\mathscr{T}$ and $g$ are given, $\mathscr{T} \subset \mathbf{R}^{N}$ is closed. Under very general assumptions we show that there exists at most one pair $(U, \Omega)$ such that $U$ is continuous

Received by the editors December 7, 1988 and, in revised form, April 17, 1989. This paper was presented at the conferences "I Reunion Hispano-Italiana de Analisis no Lineal y Matematica Aplicada", El Escorial (Spain), June 5-9, 1989, and "Workshop on Control Theory", Pisa (Italy), July $17-18,1989$.

1980 Mathematics Subject Classification (1985 Revision). Primary 35F20, 49C20, 90D25. 
in $\Omega \backslash \mathscr{T}$ and up to $\partial \mathscr{T}, \Omega \supset \mathscr{T}$ is open, and the boundary value problem is satisfied in the viscosity sense. This says that $\partial \Omega$ has to be considered as a free boundary, whose determination is part of the problem which is to be solved. We also give some sufficient conditions under which such a solution pair exists. This result bears some similarities with one due to Giusti [26] for the equation of surfaces of prescribed mean curvature.

The second problem we consider is the local uniqueness around $\partial \mathscr{T}$ of solutions to

$$
\begin{cases}H(x, D U)=0 & \text { in } \mathscr{O} \backslash \mathscr{T}, \\ U=g & \text { on } \partial \mathscr{T},\end{cases}
$$

for some open set $\mathscr{O}$ having nonempty intersection with the closed set $\mathscr{T}$. We prove this under a one-sided condition involving the solutions to compare and the boundary data $g$, but with no assumptions on $\mathscr{T}$.

The whole work is motivated by the theory of optimal control and differential games and we use both viscosity solutions methods and differential games methods. Indeed our uniqueness results are obtained by giving a representation formula for the solution $U$ as the value function of a differential game with dynamics $y^{\prime}=f(y, a, b)$, running cost $h$, terminal set $\mathscr{T}$, and terminal cost $g$. Also the set $\Omega$ in (0.4) can be characterized in terms of differential games and turns out to be independent of $h$ and $g$. Throughout the paper we will discuss the game theoretic meaning of most assumptions and results. We just mention here that the crucial hypothesis (0.3), saying that the running cost is always positive, is typical of a class of important applied problems, including the min-max capture time in pursuit-evasion games and the classical minimal time problem in control theory.

Several applications of our PDE results to the theory of control and games are given in $\S 7$. In particular we prove a rather general relaxation theorem (which can be viewed as a weak form of the bang-bang principle) which to our knowledge is the first one holding for differential games.

The boundary value problem $(0.4)$ was first studied in [4] as the natural one for the minimal time function ( $h \equiv 1, g \equiv 0, B$ a singleton), and in [7] for pursuit-evasion games. However both these papers consider $\partial \Omega$ as a given fixed boundary and (0.4) as a Dirichlet problem. The idea that $\Omega$ and $U$ could in fact be determined simultaneously has been pointed out by Falcone and one of the authors in [5], where the convergence of a discrete approximation to the minimal time function is studied.

The local uniqueness of positive solutions of (0.5) was discovered by EvansJames [22] for the minimal time problem with $\mathscr{T}=\{0\}$, and our result follows their ideas. The interest of this problem for the synthesis of optimal controls has been pointed out by Hermes [28].

The paper is organized as follows. In $\S 1$ we recall all the necessary definitions and notations of game theory and recall the connection with viscosity solutions of the Bellman-Isaacs PDE. We introduce Kruzkov's change of variable [36] 
$u(x)=1-\exp (-U(x))$, which is fundamental in the whole paper and which transforms $(0.4)$ into a problem in all of $\mathbf{R}^{N} \backslash \mathscr{T}$. Finally, we discuss briefly the possibility of representing a Hamiltonian in the form (0.2), (0.3) (Remark 1.5). In $\S 2$ we prove some preliminary results on Dirichlet problems (comparison between semicontinuous sub- and supersolutions and a representation formula) which extend the work of various authors, e.g. Ishii [31], Crandall-Ishii-Lions [18], Evans-Ishii [21], and which are of independent interest. In $\S 3$ we state and prove the representation formula for the solution pair of $(0.4)$. $\S 4$ deals with the local uniqueness of solutions to $(0.5)$. In $\S 5$ we take a smooth $\mathscr{T}$, introduce a condition on the directions of the family of vector fields $f$ on $\partial \mathscr{T}$, and show that these assumptions imply the local Lipschitz continuity of the solution to $(0.4)$. In $\S 6$ we put together results from all the previous sections to prove the existence of solutions to $(0.4)$. $\S 7$ contains miscellaneous remarks on applications and related problems, e.g. the regularity of the free boundary, discontinuous solutions and so on. A more complete summary of the contents can be found at the beginning of each section.

The theory of viscosity solutions of $\mathrm{HJ}$ equations was initiated by M. Crandall and P. L. Lions [19], see also Crandall-Evans-Lions [17] and P. L. Lions [38] where the connection with control theory was first remarked. The extension to possibly discontinuous solutions starts with the work of Ishii [32, 34] and Barles-Perthame [8]. The recent paper of Crandall-Ishii-Lions [18] provides some very general comparison results and an updated list of references. There are by now many papers which apply this theory to control problems: see the references in [4] and e.g. Capuzzo Dolcetta-Ishii [15], Cannarsa-Soner [14], Soner [42]. Applications to the theory of differential games have been studied by Barron-Evans-Jensen [9], Evans-Souganidis [23], Evans-Ishii [21], Lions-Souganidis [39], Souganidis [43], Berkovitz [11, 12], Subbotina-SubbotinTret' jakov [44], Subbotin-Tarasyev [45].

For the classical theory of differential games see Isaacs [30], Fleming [24], Friedman [25], Hajek [27], Elliott-Kalton [20], Krassovski-Soubbotine [35], Basar-Olsder [10].

\section{DEFINITIONS, NOTATIONS AND PRELIMINARY RESUltS}

We begin by recalling the definition of the value for a class of differential games following Elliott-Kalton [20]. We consider the system in $\mathbf{R}^{N}$, controlled by two players,

$$
y(t)=x+\int_{0}^{t} f(y(s), a(s), b(s)) d s,
$$

where $a$ and $b$ belong respectively to the following sets of admissible controls:

$$
\mathscr{A}:=\left\{a: \mathbf{R}_{+} \rightarrow A \text { measurable }\right\}, \quad \mathscr{B}:=\left\{b: \mathbf{R}_{+} \rightarrow B \text { measurable }\right\},
$$

and $A, B \subset \mathbf{R}^{M}$ are two given sets. A solution of this system for given $a$ and $b$ will be indicated by $y_{x}(\cdot)$ or $y(\cdot)$. Consider the target set $\mathscr{T} \subset \mathbf{R}^{N}$ and 
define

$$
t_{x}=t_{x}(a, b):=\inf \{t: y(t) \in \mathscr{T}\} \leq+\infty,
$$

where $t_{x}=+\infty$ if $y_{x}(\cdot)$ never reaches the target. Let the payoff of the game be

$$
P(x, a, b):=\int_{0}^{t_{x}} h\left(y_{x}(t), a(t), b(t)\right) d t+g\left(y_{x}\left(t_{x}\right)\right) .
$$

The set of admissible strategies for the first player is

$$
\begin{aligned}
\Delta:=\{\alpha: \mathscr{B} \rightarrow \mathscr{A} & : b(t)=b^{\prime}(t) \text { for all } t \leq t^{\prime} \\
& \text { implies } \left.\alpha[b](t)=\alpha\left[b^{\prime}\right](t) \text { for all } t \leq t^{\prime}\right\} .
\end{aligned}
$$

The (lower) value of the game is defined by

$$
V(x):=\inf _{\alpha \in \Delta} \sup _{b \in \mathscr{B}} P(x, \alpha[b], b) .
$$

In the special case when $h \equiv 1$ and $g \equiv 0, V$ is the capture time

$$
T(x):=\inf _{\alpha \in \Delta} \sup _{b \in \mathscr{B}} t_{x}(\alpha[b], b) .
$$

The following assumptions will hold throughout the paper:

$$
\left\{\begin{array}{l}
A \text { and } B \text { are compact } ; \mathscr{T} \text { is closed; } \\
f: \mathbf{R}^{N} \times A \times B \rightarrow \mathbf{R}^{N} ; h: \mathbf{R}^{N} \times A \times B \rightarrow \mathbf{R} \text { are continuous; } \\
|f(x, a, b)-f(y, a, b)| \leq L|x-y| ; h(x, a, b) \geq 1 ; \\
g: \mathscr{T} \rightarrow[G ;+\infty[\text { is continuous. }
\end{array}\right.
$$

We remark that if $h(x, a, b) \geq h_{0}>0$ we can rescale the problem in order to get $h_{0}=1$. We note that $(\mathrm{H} 1)$ implies $V(x) \geq G$. Moreover, we can choose $K>0$ and $\omega: \mathbf{R}_{+} \times \mathbf{R}_{+} \rightarrow \mathbf{R}_{+}$such that $\omega(\cdot, R)$ is continuous, increasing, $\omega(0, R)=0$, and we have the estimates

$$
\begin{cases}|f(x, a, b)| \leq K(1+|x|) & \text { for all } x, a, b, \\ |h(x, a, b)-h(y, a, b)| \leq \omega(|x-y|, R) & \text { whenever }|x|,|y| \leq R \\ & \text { and for all } a, b .\end{cases}
$$

We now define the (lower) capturability set, namely the set of starting points of the game such that the first player has a strategy which forces the system into the target in finite time, no matter what control the second player chooses, i.e.

$$
\mathscr{R}:=\{x: T(x)<+\infty\} .
$$

Lemma 1.1. Assume (H1). Then $\mathscr{R}=\{x: V(x)<+\infty\}$.

Proof. The estimate $V(x) \geq T(x)+G$ implies $\mathscr{R} \supset\{x: V(x)<+\infty\}$. On the other hand if $x \in \mathscr{R}$ there exists a strategy $\alpha_{0}$ such that for all $b \in \mathscr{B}$ we have $t_{x}\left(\alpha_{0}[b], b\right) \leq C_{1}$. Then by Gronwall's Lemma

$$
\left|y_{x}(t)\right| \leq C_{2} \text { for all } t \leq t_{x}, \quad b \in \mathscr{B},
$$

which implies $P\left(x, \alpha_{0}[b], b\right) \leq C_{3}$ for all $b \in \mathscr{B}$. 
Now we define the Kruzkov transform of the value function by setting $\psi(r)=$ $1-\exp (-r)$, and

$$
v(x):= \begin{cases}\psi(V(x)) & \text { if } x \in \mathscr{R}, \\ 1 & \text { elsewhere. }\end{cases}
$$

It is important to remark that $v$ is bounded and it is itself the value function of a differential game, namely

$$
v(x):=\inf _{\alpha \in \Delta} \sup _{b \in \mathscr{B}} \psi(P(x, \alpha[b], b)),
$$

where the payoff is

$$
\begin{aligned}
& \psi(P(x, a, b))= \int_{0}^{t_{x}} h\left(y_{x}(t), a(t), b(t)\right) \\
& \cdot \exp \left(-\int_{0}^{t} h\left(y_{x}(s), a(s), b(s)\right) d s\right) d t \\
&+\exp \left(-\int_{0}^{t_{x}} h\left(y_{x}(s), a(s), b(s)\right) d s\right) \psi\left(g\left(y_{x}\left(t_{x}\right)\right)\right) .
\end{aligned}
$$

We are now going to associate a Hamilton-Jacobi equation to the differential game. We recall Ishii's extension to discontinuous functions of the definition of viscosity solution [32]. Let $F: \Omega \times \mathbf{R} \times \mathbf{R} \rightarrow \mathbf{R}^{N}, \Omega \subset \mathbf{R}^{N}$, and $u_{1}, u_{2}: \Omega \rightarrow \mathbf{R}$ be respectively an upper and a lower semicontinuous function. $u_{1}, u_{2}$ are respectively a viscosity subsolution and supersolution of $F(x, u, D u)=0$ in $\Omega$ if for all $\varphi \in C^{1}(\Omega)$ such that $u_{1}-\varphi$ has a local maximum at $y$, respectively $u_{2}-\varphi$ has a local minimum at $y$, we have $F\left(y, u_{1}(y), D \varphi(y)\right) \leq 0$, and respectively $F\left(y, u_{2}(y), D \varphi(y)\right) \geq 0$. For a function $u: \Omega \rightarrow \mathbf{R}$, the upper and lower semicontinuous envelopes are defined by

$$
u^{*}(x):=\limsup _{y \rightarrow x} u(y), \quad u_{*}(x):=\liminf _{y \rightarrow x} u(y) .
$$

$u$ is a viscosity solution of $F(x, u, D u)=0$ in $\Omega$ if $u^{*}$ is a subsolution and $u_{*}$ is a supersolution.

The relevant Hamiltonians for our problem are

$$
H(x, p):=\min _{b \in B} \max _{a \in A}\{-f(x, a, b) \cdot p-h(x, a, b)\},
$$

and

$$
\mathscr{H}(x, r, p):=\min _{b \in B} \max _{a \in A}\{-f(x, a, b) \cdot p-h(x, a, b)+(h(x, a, b)-1) r\} .
$$

Lemma 1.2. Assume (H1). Then the following conditions are satisfied by $F=H$, $\mathscr{H}$ :

$$
\begin{aligned}
F & : \mathbf{R}^{N} \times \mathbf{R} \times \mathbf{R} \rightarrow \mathbf{R}^{N} \quad \text { is continuous } \\
r & \rightarrow F(x, r, p) \quad \text { is nondecreasing for all } x, p ; \\
|F(x, r, p)-F(y, r, q)| & \leq K(1+|x|)|p-q|+|q| L|x-y|+\rho(|x-y|, R)
\end{aligned}
$$


for all $x, y, p, q \in \mathbf{R}^{N},|x|,|y| \leq R,|r| \leq R, R>0$, with $\rho$ sharing the properties of $\omega$ in (1.2).

Proof. Define $l(x, a, b):=h(x, a, b)-1$. Fix $b^{\prime} \in B$ such that for all $a$

$$
\mathscr{H}(y, r, q) \geq-f\left(y, a, b^{\prime}\right) \cdot q-h\left(y, a, b^{\prime}\right)+l\left(y, a, b^{\prime}\right) r .
$$

Now fix $a^{\prime}$ such that

$$
\mathscr{H}(x, s, p) \leq-f\left(x, a^{\prime}, b^{\prime}\right) \cdot p-h\left(x, a^{\prime}, b^{\prime}\right)+l\left(x, a^{\prime}, b^{\prime}\right) s .
$$

Using (H1) and (1.2) we get

$$
\begin{aligned}
\mathscr{H}(x, s, p)-\mathscr{H}(y, r, q) \leq & K(1+|x|)|p-q|+|q| L|x-y|+\omega(|x-y|, R) \\
& +|r| \omega(|x-y|, R)+\sup _{a ; b} l(x, a, b)(s-r) .
\end{aligned}
$$

This implies (1.5) and (1.6) with $\rho(s, R)=(1+R) \omega(s, R)$. The proof for $H$ is the same.

Proposition 1.3. Assume (H1).

(i) If $\mathscr{R}$ is open and $V$ is locally bounded, then $V$ is a viscosity solution of

$$
H(x, D V)=0 \quad \text { in } \mathscr{R} \backslash \mathscr{T} \text {. }
$$

(ii) $v$ is a viscosity solution of

$$
v+\mathscr{H}(x, v, D v)=0 \quad \text { in } \mathbf{R}^{N} \backslash \mathscr{T} .
$$

Proof. The proof is based on the dynamic programming principle and can be obtained by combining the arguments in [23] and [34].

Remark 1.4. It is also easy to check, using e.g. Lemma 5 in [4] and the definition of viscosity solution, that any solution of $H(x, D W)=0$ in $\Omega$, where $\Omega$ is open, is such that $w=\psi(W)$ is a solution of $w+\mathscr{H}(x, w, D w)=0$ in $\Omega$.

Remark 1.5. We have not been able to characterize the Hamiltonians which admit a representation like (1.4) with $h$ positive, while, without this sign restriction, there are very general results by Evans-Souganidis [23] and Ishii [33]. We can just prove that if a Hamiltonian is of the form

$$
F(x, p)=\phi(x, p)-h^{\prime}(x)
$$

with $\phi$ satisfying

$$
\left\{\begin{array}{l}
\phi(x, p)-\phi(x, q) \leq K(1+|x|)|p-q|, \\
\phi(x, \lambda p)=\lambda \phi(x, p) \text { for all } \lambda \geq 0 ; x, p, q \in \mathbf{R}^{N}, \\
|\phi(x, p)-\phi(y, p)| \leq L|x-y| \text { for all } x, y \in \mathbf{R}^{N} ;|p|=1,
\end{array}\right.
$$

then it can be represented in the form (1.4) with $h=h^{\prime}$ and suitable $A, B$, and $f$ satisfying (H1) (see Lemma 5.4 in [23]). Vice-versa any Hamiltonian of the form (1.4) with $h$ independent of $a, b$ and satisfying (H1) can be written in the form (1.9) with $\phi$ satisfying (1.10). 
We end this section by introducing two more notations:

$$
B(x, r):=\left\{y \in \mathbf{R}^{N}:|x-y|<r\right\},
$$

and for $X \subset \mathbf{R}^{N}$ and $\eta>0$

$$
B(X, \eta):=\{x: \operatorname{dist}(x, X)<\eta\} .
$$

\section{COMPARISON OF SEMICONTINUOUS SUB- AND SUPERSOLUTIONS}

In this section we prove a comparison result between bounded sub- and supersolutions of the Dirichlet problem for the equation

$$
u+F(x, u, D u)=0 \text { in } \mathscr{O},
$$

where $\mathscr{O}$ is an arbitrary open set and $F$ satisfies the structural conditions (1.5), (1.6). To do this we implement the program of Crandall-Ishii-Lions [18] by a suitable choice of test functions which follows [4]. Here however we follow a suggestion of one of the referees of [4] and introduce a modification inspired by Ishii [31] which allows us to assume that the functions to compare are just continuous on $\partial \mathscr{O}$, instead of uniformly continuous as in [4]. The comparison theorems provide both uniqueness and continuity of viscosity solutions as soon as they are continuous on the boundary. This will be exploited in the existence theory in $\S 6$ and at the end of the present section to generalize a representation formula of Evans-Ishii [21] which we will need in $\S \S 3-4$.

Lemma 2.1. Assume $F$ satisfies (1.6). Then $F$ satisfies the following property (P): There exists a Lipschitz continuous everywhere differentiable function $\mu: \mathbf{R}^{N} \rightarrow \mathbf{R}_{+}$and a constant $C$ such that

$$
F(x, r, p)-F(x, r, p+\lambda D \mu(x)) \leq \lambda C \text { for all } x \in \mathscr{O}, \lambda \in[0,1], r \in \mathbf{R},
$$

and $\lim _{|x| \rightarrow+\infty} \mu(x)=+\infty$.

Proof. As in the proof of Theorem 2 in [4] we can take

$$
\mu(x):= \begin{cases}k(|x|) & \text { if }|x|<e, \\ \log (|x|) & \text { if }|x| \geq e,\end{cases}
$$

where $k \in C^{1}(\mathbf{R})$ satisfies $k(0)=k^{\prime}(0)=0, k(e)=1$, and $k^{\prime}(e)=1 / e$.

Lemma 2.2. Let $\Omega$ be an open set in $\mathbf{R}^{n}$, and let $F^{\wedge}: \Omega \times \mathbf{R}^{n} \rightarrow \mathbf{R}$ satisfy property $(\mathrm{P})$ in Lemma 2.1. Let $z$ be a viscosity subsolution of $z+F^{\wedge}(x, D z)=$ 0 in $\Omega$, and let $w \in C^{1}(\Omega)$ satisfy $w(x)+F^{\wedge}(x, D w) \geq 0$ and $|D w| \leq C$ for all $x \in \Omega$, such that $w(x)<z(x)$. Assume that

$$
\sup _{y \in \partial \Omega} \limsup _{\Omega \ni x \rightarrow y}(z(x)-w(x))<\sup _{\Omega}(z-w)<+\infty .
$$

Then $z \leq w$ in $\Omega$.

Proof. See Lemma 1 and Remark 3 in Crandall-Ishii-Lions [18]. 
Theorem 2.3. Suppose $F$ satisfies (1.5), (1.6) and $\mathscr{O} \subset \mathbf{R}^{N}$ is open. Let $v_{1}, v_{2}: \overline{\mathscr{O}} \rightarrow \mathbf{R}$ be respectively a viscosity sub-and supersolution of

$$
v+F(x, v, D v)=0 \text { in } \mathscr{O},
$$

and assume that they are bounded, continuous in each point of $\partial \mathscr{O}$, and $v_{1} \leq v_{2}$ on $\partial \mathscr{O}$. Then $v_{1} \leq v_{2}$ in $\mathscr{O}$.

Proof. Following [18] we consider

$$
F^{\wedge}(x, y, p, q):=F\left(x, v_{1}(x), p\right)-F\left(y, v_{2}(y),-q\right)
$$

and observe that $z(x, y):=v_{1}(x)-v_{2}(y)$ is a viscosity subsolution of

$$
z+F^{\wedge}\left(x, y, D_{x} z, D_{y} z\right)=0 \text { in } \mathscr{O} \times \mathscr{O} .
$$

For $\beta, m>0$ define $R(\beta)=((\sup z+1) / \beta)^{1 / m}$ and let $\bar{\beta}:=\bar{\beta}(m)$ be such that $R(\beta) \geq \max \left(\left\|v_{1}\right\|_{\infty},\left\|v_{2}\right\|_{\infty}\right)$ for all $\beta \leq \bar{\beta}$. Define $\sigma_{\beta}(s)=\rho(s, R(\beta))$,

$$
\begin{gathered}
\Omega:=\Omega_{\beta}:=\left\{(x, y) \in \mathbf{R}^{2 N}:|x|,|y|<R(\beta),|x-y|<\sigma_{\beta}^{-1}(\beta / 2)\right\} \cap(\mathscr{O} \times \mathscr{O}), \\
w(x, y):=w_{\varepsilon, \beta}(x, y):=\left(\left(\varepsilon^{4 L}+|x-y|^{2}\right)^{1 / 2 L}\right) / \varepsilon+\beta\left(\langle x\rangle^{m}+\langle y\rangle^{m}\right),
\end{gathered}
$$

where $\langle x\rangle:=\left(1+|x|^{2}\right)^{1 / 2}, \varepsilon>0$, and $\left.\left.m \in\right] 0,1\right]$ will be chosen later. Note that

$$
\alpha:=\alpha(\varepsilon, \beta):=\sup _{\Omega}(z-w) \leq A<+\infty \text { for all } \varepsilon, \beta>0 .
$$

If $\liminf _{(\varepsilon \beta) \rightarrow(0,0)} \alpha \leq 0$, we have for a sequence $\varepsilon_{n} \rightarrow 0, \beta_{n} \rightarrow 0$

$$
z(x, x) \leq w(x, x)+\alpha \leq \varepsilon_{n}+2 \beta_{n}\langle x\rangle^{m}+\alpha \text { for all } x \in \mathscr{O} \cap B\left(0, R\left(\beta_{n}\right)\right),
$$

which implies the desired conclusion $z(x, x) \leq 0$ for all $x \in \mathscr{O}$. Then we will assume

$$
\left.\alpha(\varepsilon, \beta) \geq \bar{\alpha}>0 \text { for all } \varepsilon, \beta \in] 0, \varepsilon_{0}\right],
$$

and show that $z$ and $w$ satisfy the assumptions of Lemma 2.2 in $\Omega$ for a sequence $\varepsilon_{n} \rightarrow 0, \beta_{n} \rightarrow 0$, therefore reaching the same conclusion as above. In the points where $z(x, y)=v_{1}(x)-v_{2}(y) \geq w \geq 0$, the monotonicity of $F$ in $r$ implies

$$
F^{\wedge}(x, y, p, q) \geq F\left(x, v_{1}(x), p\right)-F\left(y, v_{1}(x),-q\right),
$$

so that, using (1.6), we get for $(x, y) \in \Omega$,

$$
\begin{aligned}
& w(x, y)+F^{\wedge}\left(x, y, D_{x} w, D_{y} w\right) \\
& \quad \geq w(x, y)-L|x-y|^{2}\left(\left(\varepsilon^{4 L}+|x-y|^{2}\right)^{1 / 2 L-1}\right) /(\varepsilon L)-L|x-y||y| \beta m\langle y\rangle^{m-2} \\
& \quad-\rho(|x-y|, R(\beta))-K(1+|x|) m \beta\left|x\langle x\rangle^{m-2}+y\langle y\rangle^{m-2}\right| \\
& \quad \geq \beta\left(\langle x\rangle^{m}+\langle y\rangle^{m}\right)-3 m \beta(L+K)\left(\langle x\rangle^{m}+\langle y\rangle^{m}\right)-\beta / 2,
\end{aligned}
$$

which can be made $\geq 0$ choosing $m \leq[6(L+K)]^{-1}$. 
It remains to check the condition $\sup _{\partial \Omega}(z-w)<\bar{\alpha}$. Suppose by contradiction that $\left(x^{\prime}, y^{\prime}\right) \in \partial \Omega$ satisfies $z\left(x^{\prime}, y^{\prime}\right)-w\left(x^{\prime}, y^{\prime}\right) \geq \bar{\alpha} / 2>0$, and observe first of all that $\left|x^{\prime}\right|=R(\beta)$ or $\left|y^{\prime}\right|=R(\beta)$ and the definition of $R(\beta)$ imply

$$
z\left(x^{\prime}, y^{\prime}\right)-w\left(x^{\prime}, y^{\prime}\right) \leq \sup z-\beta R(\beta)^{m}=-1,
$$

a contradiction. Thus we can choose $\delta, 0<\delta<\sigma_{\beta}^{-1}(\beta / 2)$, such that $\left|x^{\prime}-y^{\prime}\right|<$ $\delta$ implies $\left|v_{i}\left(x^{\prime}\right)-v_{i}\left(y^{\prime}\right)\right|<\bar{\alpha} / 2, i=1,2$, because either $x^{\prime}$ or $y^{\prime}$, say $x^{\prime}$, belong to $\partial \mathscr{O}$ where both $v_{i}$ are continuous. This leads to a contradiction because $z\left(x^{\prime}, x^{\prime}\right) \leq 0$ implies

$$
z\left(x^{\prime}, y^{\prime}\right)-w\left(x^{\prime}, y^{\prime}\right) \leq z\left(x^{\prime}, y^{\prime}\right)-z\left(x^{\prime}, x^{\prime}\right)=v_{2}\left(x^{\prime}\right)-v_{2}\left(y^{\prime}\right)<\bar{\alpha} / 2 .
$$

On the other hand if $\left|x^{\prime}-y^{\prime}\right| \geq \delta$ we use

$$
z\left(x^{\prime}, y^{\prime}\right) \geq w_{\varepsilon, \beta}\left(x^{\prime}, y^{\prime}\right),
$$

and get a contradiction for each fixed $\beta>0$ by taking $\varepsilon$ small enough so that the right-hand side is larger than the left-hand side.

Corollary 2.4. Assume (H1). Let $\Omega \supset \mathscr{T}$ be an open set, let $v_{1}, v_{2}: \overline{\Omega \backslash \mathscr{T}} \rightarrow \mathbf{R}$ be respectively a viscosity sub-and supersolution of

$$
v+\mathscr{H}(x, v, D v)=0 \quad \text { in } \Omega \backslash \mathscr{T},
$$

and suppose they are bounded, continuous in each point of $\partial \Omega$ and $\partial \mathscr{T}$ and $v_{1} \leq v_{2}$ on $\partial \Omega \cup \partial \mathscr{T}$. Then $v_{1} \leq v_{2}$ in $\Omega \backslash \mathscr{T}$. In particular, if $v$ is continuous on $\partial \mathscr{T}$, then it is continuous in $\mathbf{R}^{N} \backslash \mathscr{T}$ and it is the unique bounded viscosity solution of

$$
\begin{cases}v+\mathscr{H}(x, v, D v)=0 & \text { in } \mathbf{R}^{N} \backslash \mathscr{T}, \\ v=\psi(g) & \text { on } \partial \mathscr{T},\end{cases}
$$

assuming the data continuously.

Proof. The first statement follows from Lemma 1.2 and Theorem 2.3. By Proposition 1.3, $v^{*}$ and $v_{*}$ are respectively a sub- and a supersolution of the equation, they are bounded because $v$ is, and they coincide on $\partial \mathscr{T}$ by the continuity of $v$ there. Then $v^{*} \leq v_{*}$ in $\mathbf{R}^{N} \backslash \mathscr{T}$, and $v$ is continuous. The last statement is now easy to prove.

We conclude this section with a representation formula for solutions of Dirichlet problems which generalizes a result of Evans-Ishii [21, Theorem 4.1].

Proposition 2.5. Assume (H1). Let $\Omega \subset \mathbf{R}^{N}$ be an open set and define

$$
\tau_{x}:=\inf \left\{t: y(t) \in \mathbf{R}^{N} \backslash \Omega\right\} \leq+\infty .
$$

If $u \in C(\bar{\Omega})$ is a bounded viscosity solution of $u+\mathscr{H}(x, u, D u)=0$ in $\Omega$, 
then

$$
\begin{aligned}
u(x)=\inf _{\alpha \in \Delta} \sup _{b \in \mathscr{B}}\left\{\int_{0}^{\tau_{x}}\right. & h\left(y_{x}(t), \alpha[b](t), b(t)\right) \\
& \cdot \exp \left(-\int_{0}^{t} h\left(y_{x}(s), \alpha[b](s), b(s)\right) d s\right) d t \\
& \left.+\exp \left(-\int_{0}^{\tau_{x}} h\left(y_{x}(s), \alpha[b](s), b(s)\right) d s\right) u\left(y_{x}\left(\tau_{x}\right)\right)\right\} .
\end{aligned}
$$

(Note that only values of $u$ on $\partial \Omega$ appear in the right-hand side.)

Proof. For $\varepsilon>0$ set $\Omega_{\varepsilon}:=\{x \in \Omega$ : $\operatorname{dist}(x, \partial \Omega)>\varepsilon,|x|<1 / \varepsilon\}$, and let $\zeta_{\varepsilon} \in C^{\infty}\left(\mathbf{R}^{N}\right)$ be such that $\zeta_{\varepsilon}=1$ on $\Omega_{\varepsilon}, \zeta_{\varepsilon}=0$ on $\mathbf{R}^{N} \backslash \Omega$, and $0 \leq \zeta_{\varepsilon} \leq 1$ everywhere. Extend $u$ to be a continuous and bounded function defined on $\mathbf{R}^{N}$. Then $u$ is a viscosity solution of

$u+\inf _{b \in B} \sup _{a \in A}\left\{-f_{\varepsilon}(x, a, b) \cdot D u-m_{\varepsilon}(x, a, b)+(h(x, a, b)-1) u\right\}=0 \quad$ in $\mathbf{R}^{N}$,

where $f_{\varepsilon}:=f \zeta_{\varepsilon}, m_{\varepsilon}:=h_{\varepsilon}+h y\left(1-\zeta_{\varepsilon}\right)$ and $h_{\varepsilon}:=h \zeta_{\varepsilon}$. Another viscosity solution of this equation is

$$
u_{\varepsilon}(x)=\inf _{\alpha \in \Delta} \sup _{b \in \mathscr{B}}\left\{\int_{0}^{\infty} m_{\varepsilon}\left(y_{x}, \alpha[b], b\right) \exp \left(-\int_{0}^{t} h\left(y_{x}, \alpha[b], b\right) d s\right) d t\right\},
$$

by the arguments of the proof of Proposition 1.3. We can apply Theorem 2.3 with $\mathscr{O}=\mathbf{R}^{N}$ and get $\left(u_{\varepsilon}\right)^{*} \leq u \leq\left(u_{\varepsilon}\right)_{*}$, that is $u=u_{\varepsilon}$. The rest of the proof is the same as in [21, Theorem 4.1]: by the dynamic programming principle [21, Theorem 3.1] and the definition of $f_{\varepsilon}, h_{\varepsilon}, m_{\varepsilon}$, one can write for all $\varepsilon>0$

$$
\begin{aligned}
& u(x)=\inf _{\alpha \in \Delta} \sup _{b \in \mathscr{B}}\left\{\int_{0}^{\tau_{x}^{\ell}} h\left(y_{x}, \alpha[b], b\right)\right. \\
& \cdot \exp \left(-\int_{0}^{t} h\left(y_{x}, \alpha[b], b\right) d s\right) d t \\
&\left.+\exp \left(-\int_{0}^{\tau_{x}^{\varepsilon}} h\left(y_{x}, \alpha[b], b\right) d s\right) u\left(y_{x}\left(\tau_{x}^{\varepsilon}\right)\right)\right\}
\end{aligned}
$$

where $\tau_{x}^{\varepsilon}$ is the first exit time from $\Omega_{\varepsilon}$, and then for each $x \in \Omega$ one shows that the last formula coincides with $(2.1)$ as $\varepsilon$ goes to 0 . For the details of this last part see [21].

\section{REPRESENTATION FORMULAS FOR THE GLOBAL PROBLEM}

The main result of this section is the following.

Theorem 3.1. Assume (H1). If there exist an open set $\Omega \supset \mathscr{T}$ and a function $U \in C(\Omega \backslash \mathscr{T})$ bounded below and continuous on $\partial \mathscr{T}$ which is a viscosity 
solution of

$$
\begin{cases}H(x, D U)=0 & \text { in } \Omega \backslash \mathscr{T}, \\ U=g & \text { on } \partial \mathscr{T}, \\ U(x) \rightarrow+\infty & \text { as } x \rightarrow x_{0} \in \partial \Omega,\end{cases}
$$

then $\Omega=\mathscr{R}$ and $U=V$.

Remark 3.2. This theorem can also be used as a comparison result alternative to Theorem 2.3. Its main advantage and novelty is that we do not assume $\Omega=\mathscr{R}$ a priori, therefore showing that the right concept of solution for the boundary value problem (3.1) is not just $U$ for given $\Omega$, but the pair $(\Omega, U)$. Observe that $\Omega=\mathscr{R}$ does not depend on $h$ and $g$.

Proof. Let $(\Omega, U)$ satisfy the hypotheses of the theorem, and $u=\psi(U)$. We observe that $u \in C(\Omega \backslash \tau)$ and by Remark 1.4 and Proposition 2.5 we have

$$
\begin{aligned}
u(x)=\inf _{\alpha \in \Delta} \sup _{b \in \mathscr{B}}\left\{\int_{0}^{\tau_{x}}\right. & h\left(y_{x}, \alpha[b], b\right) \\
& \cdot \exp \left(-\int_{0}^{t} h\left(y_{x}, \alpha[b], b\right) d s\right) d t \\
& \left.+\exp \left(-\int_{0}^{\tau_{x}} h\left(y_{x}, \alpha[b], b\right) d s\right) u\left(y_{x}\left(\tau_{x}\right)\right)\right\},
\end{aligned}
$$

with $\tau_{x}:=\inf \left\{t: y_{x}(t) \in \partial(\Omega \backslash \mathscr{T})\right\}$.

First of all we prove that $\Omega \subset \mathscr{R}$. Let $x \in \Omega \backslash \mathscr{T}$ and $0<\varepsilon<1-u(x)$. By (3.2) we can choose $\bar{\alpha} \in \Delta$ such that

$$
u(x)+\varepsilon \geq 1-\exp \left(-\int_{0}^{\tau_{x}} h(y, \bar{\alpha}[b], b) d s\right)\left(1-u\left(y\left(\tau_{x}\right)\right)\right) \text { for all } b \in \mathscr{B} .
$$

This implies $y\left(\tau_{x}\right) \in \partial \mathscr{T}$ and $\tau_{x} \leq \psi^{-1}[(u(x)+\varepsilon-\psi(G) /[1=x(G))])$ for all $b \in \mathscr{B}$. Therefore we have $x \in \mathscr{R}$.

Suppose now that there exists $x \in \mathscr{R} \cap \partial \Omega$. Fix $\varepsilon>0$ and $\bar{\alpha} \in \Delta$ such that $v(x)<1-\varepsilon$ and

$$
\psi(P(x, \bar{\alpha}[b], b))<1-\varepsilon \text { for all } b \in \mathscr{B} .
$$

Therefore choose $C_{1}>0$ such that $t_{x}(\bar{\alpha}[b], b) \leq C_{1}$ for all $b \in \mathscr{B}$ and then by Gronwall's Lemma

$$
\left|y_{x}(t)\right| \leq(1+|x|) \exp \left(K C_{1}\right)=: R \text { for all } t \leq t_{x}, \quad b \in \mathscr{B} .
$$

Let $C_{2}:=\sup _{B(0, R) \times A \times B}|f(z, a, b)|,\|\psi(g)\|_{R}:=\sup \{|\psi(g(x))|: x \in \partial \mathscr{T} \cap$ $B(0, R)\}$, and $\eta<\min \left\{\varepsilon, 1-\|\psi(g)\|_{R}\right\}$. Fix also $\delta>0$ such that $\operatorname{dist}(z, \partial \Omega)$ $\leq \delta$ and $z \in \Omega \cap B(0, R)$ imply

$$
u(z)>1-\eta>\|\psi(g)\|_{R} .
$$


For each control $b \in \mathscr{B}$ define

$$
\sigma(b):=\sup \left\{s \leq t_{x}(\bar{\alpha}[b], b): \operatorname{dist}\left(y_{x}(s), \partial \Omega\right)=\delta, y_{x}(t) \in \Omega \text { for } t \geq s\right\} .
$$

We want to prove the following statement: there exists $\bar{b} \in \mathscr{B}$ such that, setting $\sigma:=\sigma(\bar{b})$ and $\widetilde{\alpha}[b](\cdot)=\bar{\alpha}\left[b^{\prime}\right](\sigma+\cdot)$, where

we have

$$
b^{\prime}(t):= \begin{cases}\bar{b}(t), & t \leq \sigma, \\ b(t-\sigma), & t>\sigma,\end{cases}
$$

$$
t_{y(\sigma)}(\widetilde{\alpha}[b], b)=\tau_{y(\sigma)}(\widetilde{\alpha}[b], b) \quad \text { for all } b \in \mathscr{B} .
$$

In fact, if (3.5) holds, we then have, by the definition of $\sigma,(3.2),(3.3)$, and (3.4)

$$
\begin{aligned}
& 1-\eta<u(y(\sigma)) \leq \sup _{b \in \mathscr{B}}\left\{\int_{0}^{\tau_{y(\sigma)}} h(y, \tilde{\alpha}[b], b)\right. \quad \cdot \exp \left(-\int_{0}^{t} h(y, \widetilde{\alpha}[b], b) d s\right) d t \\
&\left.\quad+\exp \left(-\int_{0}^{\tau_{y(\sigma)}} h(y, \widetilde{\alpha}[b], b) d s\right) u\left(y\left(\tau_{y(\sigma)}\right)\right)\right\} \\
&= \sup _{b \in \mathscr{B}}\{\psi(P(y(\sigma), \widetilde{\alpha}[b], b))\} \\
& \leq \sup _{b \in \mathscr{B}}\{\psi(P(x, \bar{\alpha}[b], b))\}<1-\varepsilon,
\end{aligned}
$$

which gives a controdiction with the choice of $\eta$ and therefore

$$
\mathscr{R} \cap \partial \Omega=\varnothing .
$$

We suppose by contradiction that (3.5) does not hold and define for $b_{0} \in \mathscr{B}$ :

$$
\tilde{b}_{1}(t):= \begin{cases}b_{0}(t), & t \leq \sigma\left(b_{0}\right)=: t_{0}, \\ b_{1}\left(t-t_{0}\right), & t>t_{0},\end{cases}
$$

where $b_{1}$ does not verify (3.5). Then we continue recursively setting

$$
\widetilde{b}_{n+1}(t):= \begin{cases}\widetilde{b}_{n}(t), & t \leq \sigma\left(\widetilde{b}_{n}\right)=: t_{n}, \\ b_{n+1}\left(t-t_{n}\right), & t>t_{n},\end{cases}
$$

where $b_{n+1}$ does not verify (3.5). We now observe that $t_{n+1}-t_{n} \geq 2 \delta / C_{2}$ by the choice of $\bar{\alpha}$ and therefore, if $\widetilde{b}(t):=\widetilde{b}_{n}(t)$ for $t \leq t_{n}$, then $t_{x}(\bar{\alpha}[\tilde{b}], \widetilde{b})>C_{1}$, which is a contradiction.

Now by (3.6) and $\mathscr{R}=(\Omega \cap \mathscr{R}) \cup\left(\operatorname{int}\left(\Omega^{c}\right) \cap \mathscr{R}\right)$, so it is the union of two disjoint relatively open sets. But if $x \in \operatorname{int}\left(\Omega^{c}\right) \cap \mathscr{R}$ there is a continuous arc in $\mathscr{R}$ joining $x$ to $\mathscr{T} \subset \Omega \cap \mathscr{R}$, and then $\Omega \supset \mathscr{R}$. To prove $u=v$, let $x \in \mathscr{R}$ and $0<\varepsilon<1-u(x)$. By (3.2) we have for some $\bar{\alpha} \in \Delta$

$$
\begin{aligned}
1> & u(x)+\varepsilon>\int_{0}^{\tau_{x}} h(y, \bar{\alpha}[b], b) \exp \left(-\int_{0}^{t} h(y, \bar{\alpha}[b], b) d s\right) d t \\
& +\exp \left(-\int_{0}^{\tau_{x}} h(y, \bar{\alpha}[b], b) d s\right) u\left(y\left(\tau_{x}\right)\right) \quad \text { for all } b \in \mathscr{B} .
\end{aligned}
$$


Therefore $\tau_{x}<+\infty$ and $y\left(\tau_{x}\right) \in \partial \mathscr{T}$, that is

$$
\tau_{x}(\bar{\alpha}[b], b)=t_{x}(\bar{\alpha}[b], b) \text { for all } b \in \mathscr{B} .
$$

As a consequence we have

$$
u(x)+\varepsilon>\psi(P(x, \bar{\alpha}[b], b)) \text { for all } b \in \mathscr{B},
$$

which by (1.3) implies $u(x) \geq v(x)$. The proof of the opposite inequality is completely analogous, using first (1.3) and then concluding by (3.2).

The following result is implicit in the proof of Theorem 3.1.

Corollary 3.3. Assume (H1). Let $u \in C\left(\overline{\mathbf{R}^{N} \backslash \mathscr{T}}\right)$ be a viscosity solution of

$$
\begin{cases}u+\mathscr{H}(x, u, D u)=0 & \text { in } \mathbf{R}^{N} \backslash \mathscr{T}, \\ u=\psi(g) & \text { on } \partial \mathscr{T},\end{cases}
$$

$u$ bounded below. Then $\mathscr{R}=\{x: u(x)<1\} \cup \mathscr{T}$ and $u=v$.

\section{REPRESENTATION FORMULAS FOR LOCAL SOLUTIONS}

In this section we give some local uniqueness result and representation formulas for continuous solutions of $H(x, D V)=0$ in $\Omega \backslash \mathscr{T}$, where $\Omega$ is any open set having nonempty intersection with $\mathscr{T}$, and we assume the condition $U=g$ only on $\partial \mathscr{T}$ and just a lower bound on the rest of the boundary. The first result of this type has been discovered very recently by Evans-James [22] for the minimum time problem with $\mathscr{T}=\{0\}$, and our proof is a generalization of theirs. Some applications will be given in $\S \S 6$ and 7.

Theorem 4.1. Assume (H1). Let $x_{0} \in \partial \mathscr{T}$ and define $B_{r}:=B\left(x_{0}, r\right), M_{r}:=$ $\max _{B_{r} \times A \times B}|f(x, a, b)|$. Suppose $U \in C\left(\overline{B_{r} \backslash \mathscr{T}}\right)$ is a viscosity solution of

$$
\begin{cases}H(x, D U)=0 & \text { in } B_{r} \backslash \mathscr{T}, \\ U=g & \text { on } \partial \mathscr{T} \cap B_{r},\end{cases}
$$

such that

$$
g\left(x_{0}\right)<r / M_{r}+\min _{\partial\left(B_{r} \backslash \mathscr{T}\right)} U .
$$

Define $\sigma(s):=\sup \left\{\left|U(x)-U\left(x_{0}\right)\right|: x \in B_{s} \backslash \mathscr{T}\right\}, s \leq r$. Then any other continuous function satisfying (4.1), (4.2) coincides with $U$ in $B_{r^{\prime}} \backslash \mathscr{T}$ for each $r^{\prime}$ such that

$$
\sigma\left(r^{\prime}\right)+r^{\prime} / M_{r} \leq r / M_{r}+\min _{\partial\left(B_{r} \backslash \mathscr{T}\right)} U-g\left(x_{0}\right) .
$$

\section{If moreover}

$$
g(x) \geq \min _{\partial\left(B_{r} \backslash \mathscr{T}\right)} U \text { for all } x \in \partial \mathscr{T}
$$

then

$$
U(x)=V(x) \text { for all } x \in B_{r^{\prime}} \backslash \mathscr{T} \text {. }
$$


Remark 4.2. The first statement is purely local and it holds for $f, h, g, \mathscr{T}$ defined only in $\bar{B}_{r}$. Then one can get a representation formula of the form (4.5) for every extension of $f, h, g, \mathscr{T}$ out of $\overline{B_{r}}$ which verifies (H1) and (4.4).

Proof. Replacing $U$ by $U-\min _{\partial\left(B_{r} \backslash \mathscr{T}\right)} U$ we can assume without loss of generality that $\min _{\partial\left(B_{r} \backslash \mathscr{G}\right)} U=0$. We will prove the second statement; the first statement will then follow substituting $\mathscr{T}$ with $\partial \mathscr{T} \cap \overline{B_{r}}$ if necessary. We also drop the subscript $r$ of $M_{r}$. Let $u=\psi(U)$. Then by Proposition 1.3 and Remark $1.4 u$ is a viscosity solution of

$$
\begin{cases}u+\mathscr{H}(x, u, D u)=0 & \text { in } B_{r} \backslash \mathscr{T} \\ u=\psi(g) & \text { on } \partial \mathscr{T} .\end{cases}
$$

Therefore by Proposition 2.5

$$
\text { (4.6) } \begin{aligned}
u(x)=\inf _{\alpha \in \Delta} \sup _{b \in \mathscr{B}}\left\{\int_{0}^{\tau_{x}}\right. & h\left(y_{x}, \alpha[b], b\right) \\
& \cdot \exp \left(-\int_{0}^{t} h\left(y_{x}, \alpha[b], b\right) d s\right) d t \\
& \left.+\exp \left(-\int_{0}^{\tau_{x}} h\left(y_{x}, \alpha[b], b\right) d s\right) u\left(y_{x}\left(\tau_{x}\right)\right)\right\},
\end{aligned}
$$

where $\tau_{x}:=\inf \left\{t: y_{x}(t) \in \partial\left(B_{r} \backslash \mathscr{T}\right)\right\}$. We have to prove that $u(x)=v(x)$ for all $x \in B_{r^{\prime}} \backslash \mathscr{T}$. We set

$$
\tau_{x ; z}(a, b):=\inf \left\{t: y_{x}(t)=z\right\} \text { and } p(s)=\sup \left\{u(x): x \in B_{s} \backslash \mathscr{T}\right\} .
$$

We observe that by (4.2) $p(0)=\left|\psi\left(g\left(x_{0}\right)\right)\right|<\psi(r / M)$ and therefore for some $\bar{\delta}<1$ we have $p(0)<\delta \psi(\delta r / M)$ for all $\bar{\delta} \leq \delta<1$. Let $r^{\prime}$ satisfy (4.3) as a strict inequality and choose $\delta \in[\bar{\delta}, 1[$ such that

$$
p\left(r^{\prime}\right) \leq \delta \psi\left(\delta\left(r-r^{\prime}\right) / M\right) \text {. }
$$

Now suppose that $x \in \overline{B_{r^{\prime}} \backslash \mathscr{T}}, z \in \partial B_{r} \backslash \mathscr{T}$, and $\tau_{x, z}(a, b)<+\infty$. Therefore we have

and then

$$
z-x=\int_{0}^{\tau_{x ; z}} f\left(y_{x}(t), a(t), b(t)\right) d t
$$

$$
\left(r-r^{\prime}\right) / M \leq \tau_{x ; z}(a, b) .
$$

Let

$$
0<2 \varepsilon<(1-\delta) \psi\left(\delta\left(r-r^{\prime}\right) / M\right) .
$$

By (4.6) we fix $\alpha_{1} \in \Delta$ such that

$$
\begin{aligned}
\int_{0}^{\tau_{x}} h\left(y_{x}, \alpha[b], b\right) \exp \left(-\int_{0}^{t} h\left(y_{x}, \alpha_{1}[b], b\right) d s\right) d t \\
\quad+\exp \left(-\int_{0}^{\tau_{x}} h\left(y_{x}, \alpha_{1}[b], b\right) d s\right) u\left(y_{x}\left(\tau_{x}\right)\right) \leq u(x)+\varepsilon \quad \text { for all } b \in \mathscr{B},
\end{aligned}
$$


and therefore, using $h \geq 1, u \geq 0,(4.7)$, and (4.9) we get

$$
\psi\left(\tau_{x}\right)+\varepsilon \leq \psi\left(\delta\left(r-r^{\prime}\right) / M\right) .
$$

If we now suppose that $z=y_{x}\left(\tau_{x}\right) \in \partial B_{r} \backslash \mathscr{T}$ for some $b_{1}$, then by (4.8)

$$
\tau_{x}\left(\alpha_{1}\left[b_{1}\right], b_{1}\right)<\delta\left(r-r^{\prime}\right) / M<\tau_{x ; z}\left(\alpha_{1}\left[b_{1}\right], b_{1}\right)
$$

and we find a contradiction. So we have proved that $\tau_{x}\left(\alpha_{1}[b], b\right)=t_{x}\left(\alpha_{1}[b], b\right)$ for all $b \in \mathscr{B}$, and therefore by (4.10)

$$
\psi\left(P\left(x, \alpha_{1}[b], b\right)\right) \leq u(x)+\varepsilon \text { for all } b \in \mathscr{B},
$$

which implies $v(x) \leq u(x)$ for all $x \in \overline{B_{r^{\prime}} \backslash \mathscr{T}}$.

To prove the equality we suppose that $v\left(x_{1}\right)<u\left(x_{1}\right)$ for $x_{1} \in\left(B_{r^{\prime}} \backslash \mathscr{T}\right)$. Then for $\eta>0$ and $\alpha_{2} \in \Delta$ we have

$$
\psi\left(P\left(x_{1}, \alpha_{2}[b], b\right)\right)+\eta<u\left(x_{1}\right) \text { for all } b \in \mathscr{B} \text {. }
$$

Thus $\tau_{x} \leq t_{x}, \psi(g) \geq 0, h \geq 1$, and (4.7) give $\psi\left(\tau_{x}\right)+\eta<\psi\left(\delta\left(r-r^{\prime}\right) / M\right)$. Again as before we deduce that

$$
\tau_{x}\left(\alpha_{2}[b], b\right)=t_{x}\left(\alpha_{2}[b], b\right) \text { for all } b \in \mathscr{B} .
$$

Therefore (4.11) implies a contradiction and we can conclude that $u=v$ in $B_{r} \backslash \mathscr{T}$.

The next theorem is an immediate consequence of Theorem 4.1.

Theorem 4.3. Assume (H1). Suppose there is an open set $\Omega \supset \mathscr{T}$ and $U \in$ $C(\overline{\Omega \backslash \mathscr{T}}), U$ bounded below, which is a viscosity solution of

$$
\begin{cases}H(x, D U)=0, & \text { in } \Omega \backslash \mathscr{T}, \\ U=g & \text { on } \partial \mathscr{T} .\end{cases}
$$

Define $d(x):=\operatorname{dist}(x, \partial \Omega), M(x):=\sup \{|f(z, a, b)|:(z, a, b) \in B(x, d(x))$ $\times A \times B\}$, and $\sigma_{x}(s):=\sup \{|U(z)-U(x)|: z \in B(x, s) \backslash \mathscr{T}\}, s \leq d(x)$. Assume that

$$
g(x)<d(x) / M(x)+\inf _{\Omega \backslash \mathscr{T}} U \text { for all } x \in \partial \mathscr{T} .
$$

Then there exists an open set $\Omega^{\prime}, \mathscr{T} \subset \Omega^{\prime} \subset \Omega$, such that $U(x)=V(x)$ for all $x \in \Omega^{\prime} \backslash \mathscr{T}$. Moreover $\operatorname{dist}\left(x, \partial \Omega^{\prime}\right) \geq r$ for all $r$ satisfying

$$
\sigma_{x}(r)+r / M(x) \leq d(x) / M(x)+\inf _{\Omega \backslash \mathscr{T}} U-g(x) .
$$

Remark 4.4. Note that if $g \equiv 0$ on $\partial \mathscr{T}$ and $U \geq 0$, then conditions (4.2) and (4.4) in Theorem 4.1 and (4.12) in Theorem 4.3 are automatically satisfied. This is the case, for instance, in time-optimal control and pursuit-evasion or survival games. If one drops condition (4.12), then the conclusion of Theorem 4.3 is false (see the example in Remark 4 of [4]). 


\section{CONTROLLABILITY AND CAPTURABILITY CONDITIONS AND REGULARITY OF $V$}

In this section we prove a regularity result for the value function $V$ which generalizes those obtained by Bardi-Falcone [5] for the minimum time function with a bounded target and by the authors [7] for the capture time. We consider a capturability condition on the vector field $f$ on the boundary of the target and show that it is necessary and sufficient for the Lipschitz continuity of the capture time $T$. Under this condition we prove that $V$ is continuous and moreover that it is locally Lipschitz if $h$ and $g$ are themselves locally Lipschitz in the space variables uniformly on the controls. Some regularity of the boundary of the target is also needed. More precisely we consider the following hypotheses:

$$
\begin{aligned}
& \mathscr{T} \text { is the closure of an open set with } C^{2} \text { boundary, } \\
& \text { for all } x \in \partial \mathscr{T} \text {, there exists } a_{x} \in A \text { such that } f\left(x, a_{x}, b\right) \cdot \\
& n(x)<0 \text { for all } b \in \mathscr{B} \text {; where } n(x) \text { is the exterior normal to } \\
& \mathscr{T} \text { at } x \text {. }
\end{aligned}
$$

Similar conditions have been considered by Fleming [24], Friedman [25] and Hajek [27]. (H3) is the counterpart for smooth targets and games of the classical "positive basis condition" in control theory due to Petrov [41]. This analogy is made precise in $[5, \S 5]$.

Lemma 5.1. Assume ( $\mathrm{H} 1)-(\mathrm{H} 3)$. Then

(i) for all $R>0$ there exist $\delta_{R}, C_{R}>0$ such that

$$
T(x) \leq C_{R} d(x) \text { for all } x \in B\left(\mathscr{T}, \delta_{R}\right) \cap B(0, R),
$$

where $d(x)=\operatorname{dist}(x, \partial \mathscr{T})$.

(ii) $\mathscr{R}$ is open.

Proof. (i) We observe that by a compactness argument for all $R>0$ there exist $\xi_{R}>0$ and $0<\delta_{R}<\xi_{R} /(2 K) \log (1+R)$ such that

$$
f\left(x, a_{x}, b\right) \cdot D d(x) \leq-\xi_{R} \text { for all } b \in B, x \in B\left(\mathscr{T}, \delta_{R}\right) \cap B(0, R) .
$$

Then we proceed with the same proof as that of Proposition 1, part (i) in [7] to get the conclusion with $C_{R}=2 / \xi_{R}$.

(ii) As in Proposition 1 of [7].

We now prove the regularity result.

Proposition 5.2. Assume (H1)-(H3). Then $V$ is continuous in $\mathscr{R} \backslash \mathscr{T}$.

Proof. Let $x_{0} \in \mathscr{R}$ and by (ii) in Lemma 5.1 we choose $\eta>0$ such that $B\left(x_{0}, \eta\right) \subset \mathscr{R}$. Let $x \in B\left(x_{0}, \eta\right)$; suppose first that $V(x) \geq V\left(x_{0}\right)$. We now denote $N:=\left(2+\left|x_{0}\right|\right) \exp \left(K\left(V\left(x_{0}\right)-G+1\right)\right), \delta_{N}, C_{N}$ as in Lemma 5.1, and $\zeta\left(x_{0}\right)=\exp \left(L\left(V\left(x_{0}\right)-G+\varepsilon\right)\right), 0<\varepsilon<1$. We can suppose that $\eta \leq \delta_{N} / \zeta\left(x_{0}\right)$. Choose $\bar{\alpha} \in \Delta$ such that

$$
\int_{0}^{t_{x_{0}}} h\left(y_{x_{0}}, \bar{\alpha}[b], b\right) d t+g\left(y_{x_{0}}\left(t_{x_{0}}\right)\right) \leq V\left(x_{0}\right)+\varepsilon \quad \text { for all } b \in \mathscr{B} .
$$


Then we have, by the choice of $N$,

$$
y_{x_{0}}\left(t_{x_{0}}(\bar{\alpha}[b], b)\right) \in \partial \mathscr{T} \cap B(0, N),
$$

and

$$
t_{x_{0}}(\bar{\alpha}[b], b) \leq V\left(x_{0}\right)+\varepsilon-G \text { for all } b \in \mathscr{B} .
$$

Redefining $\bar{\alpha}[b]$ after $t_{x_{0}}(\bar{\alpha}[b], b)$ if necessary, we can use (H2) and (H3) as in $[5,7]$ to get

$$
\left|t_{x_{0}}(\bar{\alpha}[b], b)-t_{x}(\bar{\alpha}[b], b)\right| \leq C_{N} \zeta\left(x_{0}\right)\left|x-x_{0}\right|+\varepsilon \text { for all } b \in \mathscr{B} .
$$

We also have by Gronwall's Lemma that

$$
\left|y_{x_{0}}\left(t_{x_{0}}\right)-y_{x}\left(t_{x}\right)\right| \leq K_{1} \zeta\left(x_{0}\right)\left|x-x_{0}\right| \text {. }
$$

It is easy to see that (5.1) and (5.2) imply

$$
\begin{array}{rl}
\int_{0}^{t_{x}} & h\left(y_{x}, \bar{\alpha}[b], b\right) d t+g\left(y_{x}\left(t_{x}\right)\right) \\
\leq & \int_{0}^{t_{x_{0}}} h\left(y_{x_{0}}, \bar{\alpha}[b], b\right) d t+g\left(y_{x_{0}}\left(t_{x_{0}}\right)\right) \\
& +\left(V\left(x_{0}\right)+\varepsilon-G\right) \omega\left(\zeta\left(x_{0}\right)\left|x-x_{0}\right|, N \zeta\left(x_{0}\right)\right) \\
& +\omega_{g}\left(K_{2} \zeta\left(x_{0}\right)\left|x-x_{0}\right|, N K_{2} \zeta\left(x_{0}\right)\right)+K_{3} \zeta\left(x_{0}\right)\left|x-x_{0}\right|+\varepsilon
\end{array}
$$

for all $b \in \mathscr{B}$ and $\varepsilon>0$, where $\omega_{g}(\cdot, R)$ is the modulus of continuity of $g$ in $\mathscr{T} \cap B(0, R)$. Thus

$$
\begin{aligned}
V(x) \leq & V\left(x_{0}\right)+\left(V\left(x_{0}\right)-G\right) \omega\left(\zeta\left(x_{0}\right)\left|x-x_{0}\right|, N \zeta\left(x_{0}\right)\right) \\
& +\omega_{g}\left(K_{2} \zeta\left(x_{0}\right)\left|x-x_{0}\right|, N K_{2} \zeta\left(x_{0}\right)\right)+K_{3} \zeta\left(x_{0}\right)\left|x-x_{0}\right| .
\end{aligned}
$$

Using an analogous argument in the case $V(x)<V\left(x_{0}\right)$ we can conclude the proof.

Remark 5.3. The previous proof also shows that if for all $R>0$

$|h(x, a, b)-h(y, a, b)| \leq L_{R}|x-y|$ for all $x, y \in B(0, R), a \in A, b \in B$, and

$$
|g(x)-g(y)| \leq L_{R}|x-y| \text { for all } x, y \in B(0, R),
$$

then $V$ is locally Lipschitz continuous in $\mathscr{R} \backslash \mathscr{T}$.

Remark 5.4. We can give the following local version of Proposition 5.2 by making minor changes to its proof. Assume ( $\mathrm{H} 1)$ and for $x_{0} \in \partial \mathscr{T}, r>0$, suppose that $\partial \mathscr{T} \cap B\left(x_{0}, r\right)$ is a $C^{2}$ manifold with $\mathscr{T}$ lying on one side of it and

$$
\min _{a \in A} \max _{b \in B} f(x, a, b) \cdot n(x)<0 \text { for all } x \in \partial \mathscr{T} \cap B\left(x_{0}, r\right) \text {. }
$$

Then $V$ is continuous in $B(\mathscr{T}, \delta) \cap B\left(x_{0}, r / 2\right) \backslash \mathscr{T}$ for some $\delta>0$. If moreover $h$ and $g$ satisfy (5.3) and (5.4), then $V$ is Lipschitz continuous in the same set.

We conclude this section with the following: 
Proposition 5.5. Assume (H1) and (H2). Let $1 / 2<\alpha \leq 1, \quad x_{0} \in \partial \mathscr{T}$, and suppose that $T(x) \leq C d^{\alpha}(x)$ for all $x \in B\left(x_{0}, r\right)$, where $d(x)=\operatorname{dist}(x, \partial \mathscr{T})$. Then

$$
\min _{a \in A} \max _{b \in B} f\left(x_{0}, a, b\right) \cdot n\left(x_{0}\right)<0 .
$$

Proof. It is an easy extension of the proof of [5, Theorem 5.5] and we omit it.

\section{EXISTENCE OF SOLUTIONS}

In $\S 3$ we proved that there is at most one pair $(U, \Omega)$ satisfying the boundary value problem (3.1). In the present section we will put together the results of $\S \S 2,4$ and 5 to give sufficient conditions under which the pair $(V, \mathscr{R})$ is the desired solution. The next theorem says that the whole problem reduces to the continuity of $V$ on $\partial \mathscr{T}$.

Theorem 6.1. Assume $(\mathrm{H} 1)$ and suppose that $V$ is continuous on $\partial \mathscr{T}$. Then $\mathscr{R}$ is open, $V$ is continuous in $\mathscr{R} \backslash \mathscr{T}$ and it is the unique viscosity solution bounded below and continuous on $\partial \mathscr{T}$ of

$$
\begin{cases}H(x, D V)=0 & \text { in } \mathscr{R} \backslash \mathscr{T}, \\ V=g & \text { on } \partial \mathscr{T}, \\ V(x) \rightarrow+\infty & \text { as } x \rightarrow x_{0} \in \partial \mathscr{R} .\end{cases}
$$

Proof. Since $V$ is continuous on $\partial \mathscr{T}, v$ is also. Then Corollary 2.4 applies and gives the continuity of $v$ in $\mathbf{R}^{N} \backslash \mathscr{T}$. Thus $\mathscr{R}=\{x: v(x)<1\}$ is open, and $V=-\log (1-v)$ is continuous in $\mathscr{R} \backslash \mathscr{T}$ and tends to $+\infty$ on $\partial \mathscr{R}$. By Proposition $1.3 \mathrm{~V}$ satisfies (6.1). If $U$ is another solution of $(6.1)$ then $u:=\psi(U)$ satisfies

$$
\begin{cases}u+\mathscr{H}(x, u, D u)=0 & \text { in } \mathscr{R} \backslash \mathscr{T}, \\ u=\psi(g) & \text { on } \partial \mathscr{T}, \\ u(x) \rightarrow 1 & \text { as } x \rightarrow x_{0} \in \partial \mathscr{R} .\end{cases}
$$

Thus $u=v$ by Corollary 2.4 and the proof is complete.

Remark 6.2. The fact that the continuity of $V$ on $\partial \mathscr{T}$ implies that $\mathscr{R}$ is open, $V$ is continuous, and $V \rightarrow+\infty$ on $\partial \mathscr{R}$ can be proved directly by the arguments of Proposition 5.2 and of [7, Proposition 1]. This is indeed well known for the minimal time function in control theory, as well as the equivalence between the continuity of $T$ and the property of small time local controllability (see Bacciotti [1] and Bressan [13]). This last property has been extensively studied in recent years for control systems and target $\mathscr{T}=\{0\}$ by geometric methods (see e.g. Sussmann [46], Bacciotti [3], and the references therein).

Corollary 6.3. Assume (H1)-(H3) and $g=0$. Then all the conclusions of Theorem 6.1 hold.

Proof. It suffices to recall Proposition 5.2 (and indeed Lemma 5.1 is enough). 
Corollary 6.4. Assume (H1). Suppose there exist an open set $\Omega \supset \mathscr{T}$ and $U \in$ $C(\overline{\Omega \backslash \mathscr{T}})$ which is bounded below, satisfies $H(x, D U)=0$ in $\Omega \backslash \mathscr{T}$ in the viscosity sense, coincides with $g$ on $\partial \mathscr{T}$, and verifies the compatibility condition (4.12). Then all the conclusions of Theorem 6.1 hold.

Proof. By Theorem 4.3, $V=U$ in a neighborhood of $\mathscr{T}$, which gives the desired continuity of $V$ on $\partial \mathscr{T}$.

We end this section with a local existence result which follows easily from Theorem 4.1 and Remark 5.4.

Theorem 6.5. Assume $(\mathrm{H} 1)$ and (H2). Let $\mathscr{T}_{0} \subset \partial \mathscr{T}$ be such that

$$
\min _{a \in A} \max _{b \in B} f(x, a, b) \cdot n(x)<0 \text { and } g(x)=0 \text { on } \mathscr{T}_{0} \text {. }
$$

Then for each compact $\mathscr{K} \subset \mathscr{T}_{0}$ there exist $\delta \geq \delta^{\prime}>0$ such that $V$ is a continuous viscosity solution of

$$
\begin{cases}H(x, D V)=0 & \text { in } B(\mathscr{K}, \delta) \backslash \mathscr{T}, \\ V=0 & \text { on } \mathscr{T}_{0},\end{cases}
$$

and any continuous nonnegative solution of (6.2) coincides with $V$ in $B\left(\mathscr{K}, \delta^{\prime}\right) \backslash \mathscr{T}$.

\section{APPLiCATIONS TO THE THEORY OF CONTROL AND DIFFERENTIAL GAMES}

In this section we give a number of remarks about the results of the previous sections. In particular we point out some applications to the theory of control and differential games which are often entirely new for differential games, while for control problems they are mostly just new proofs of known results. We state most of the results in the game-theoretic setting and the corresponding controltheoretic statements can be easily recovered by taking $B$ as a singleton. All the following results assume the continuity on $\partial \mathscr{T}$ of some value functions. We recall that for the minimal time function in control theory this is equivalent to small time local controllability (see Remark 6.2).

We begin by giving a sufficient condition under which two different games have the same outcome.

Theorem 7.1: Equivalence of games. Let $\mathscr{T}$ and $g$ be given and assume that the games $(f, h, A, B)$ and $\left(f^{\prime}, h^{\prime}, A^{\prime}, B^{\prime}\right)$ satisfy $(\mathrm{H} 1)$. Let $V^{\prime}$ and $\mathscr{R}^{\prime}$ be respectively the value function and the capturability set of the second game.

Suppose $V$ is continuous on $\partial \mathscr{T}$ and

$$
\begin{aligned}
\min _{b \in B} & \max _{a \in A}\{-f(x, a, b) \cdot p-h(x, a, b)\} \\
& =\min _{b \in B^{\prime}} \max _{a \in A^{\prime}}\left\{-f^{\prime}(x, a, b) \cdot p-h^{\prime}(x, a, b)\right\}
\end{aligned}
$$

for all $x, p \in \mathbf{R}^{N}$. Then $V=V^{\prime}$ and $\mathscr{R}=\mathscr{R}^{\prime}$. 
Proof. The continuity of $V$ on $\partial \mathscr{T}$ implies the continuity everywhere by Theorem 6.1. Then the conclusion follows from Theorem 3.1 because the two games have the same Hamiltonian.

If in this theorem we take $f=f, h^{\prime}=h$ and $A^{\prime} \supset A, B^{\prime} \supset B$, such that (7.1) is satisfied, then we obtain a relaxation result which can be viewed as a generalized weak version of the classical bang-bang principle. We can see this better in the following corollaries and remarks.

Corollary 7.2: Nonlinear relaxation theorem. Assume $(\mathrm{H} 1)$. Let

$$
h(x, a, b)=\bar{h}(x)
$$

and

$$
f(x, a, b)=f_{1}(x, a)+f_{2}(x, b) .
$$

Let $A^{\prime}, B^{\prime}$ be compact and such that

$$
f_{1}\left(x, A^{\prime}\right)=\operatorname{co} f_{1}(x, A), \quad f_{2}\left(x, B^{\prime}\right)=\operatorname{co} f_{2}(x, B) \quad \text { for all } x \in \mathbf{R}^{N},
$$

where $\operatorname{co} X$ indicates the closed convex hull of $X$. If either $V$ or $V^{\prime}$ is continuous on $\partial \mathscr{T}$, then $V=V^{\prime}$ and $\mathscr{R}=\mathscr{R}^{\prime}$.

Proof. We observe that

$$
\max _{q \in Q} q \cdot p=\max _{q \in \operatorname{co} Q} q \cdot p \text { and } \min _{q \in Q} q \cdot p=\min _{q \in \operatorname{co} Q} q \cdot p .
$$

Then by (7.4)

$$
\max _{a \in A^{\prime}} f_{1}(x, a) \cdot p=\max _{q \in f_{1}\left(x, A^{\prime}\right)} q \cdot p=\max _{a \in A} f_{1}(x, a) \cdot p \text { for all } p \in \mathbf{R}^{N},
$$

and

$$
\min _{b \in B^{\prime}} f_{2}(x, b) \cdot p=\min _{b \in B} f_{2}(x, b) \cdot p \quad \text { for all } p \in \mathbf{R}^{N} .
$$

Thus (7.2) and (7.3) imply (7.1).

We remark that the decoupling condition (7.3) is satisfied in the classical pursuit-evasion problem. We note also that in the special case $h \equiv 1, g \equiv 0$, we have $T=T^{\prime}$, so that

$$
\mathscr{R}(s):=\{x: T(x)<s\}=\mathscr{R}^{\prime}(s):=\left\{x: T^{\prime}(x)<s\right\} .
$$

Note that $\mathscr{R}(s)$ is the set of starting points of the game such that the first player has a strategy which forces the system into the target in time $t<s$, no matter what the second player does. $\mathscr{R}^{\prime}(s)$ has the same interpretation for the game where the controls are taken in $A^{\prime}$ and $B^{\prime}$. The corresponding objects in control theory are the sets from which one can reach $\mathscr{T}$ in time $t<s$, and therefore the conclusion (7.5) can be compared to other nonlinear relaxation theorems such as Corollary 20.2 of Hermes-LaSalle [29] and Theorem 2 of Bressan [47], which are proved by completely different methods (see also the references therein). 
Corollary 7.3: Affine relaxation theorem. Assume (H1), (7.2), and

$$
f(x, a, b)=f_{1}(x)+f_{2}(x) a+f_{3}(x) b,
$$

where $f_{i}(x), i=2,3$, are $N \times M$ matrices. If $A^{\prime}, B^{\prime}$ are compact,

$$
A^{\prime}=\operatorname{co} A, \quad B^{\prime}=\operatorname{co} B,
$$

and either $V$ or $V^{\prime}$ is continuous on $\partial \mathscr{T}$, then $V=V^{\prime}$ and $\mathscr{R}=\mathscr{R}^{\prime}$.

Proof. It is easy to see that (7.6) and (7.7) imply (7.4).

Using the remarks preceding the corollary, one can see the connection between the classical bang-bang principle of LaSalle [37] for linear control systems and Corollary 7.3.

Remark 7.4. Other sets of admissible controls. If instead of $\mathscr{A}, \mathscr{B}$ we consider as admissible controls the sets $\mathscr{A}^{\prime}, \mathscr{B}^{\prime}$ of piecewise constant controls, then one can check that the dynamic programming principle still holds and therefore the corresponding value function $V^{\prime}$ is a viscosity solution in $\mathscr{R}^{\prime} \backslash \mathscr{T}$ (see Proposition 1.3). Thus, if $V^{\prime}$ is continuous on $\partial \mathscr{T}$, by Theorems 6.1 and 3.1 we get $V=V^{\prime}$ and $\mathscr{R}=\mathscr{R}^{\prime}$, and it is not hard to see that (H2) and (H3) imply the desired continuity of $V^{\prime}$ if $g=0$. A similar result has been proved by Bacciotti [2] for linear control systems. The above argument and the same conclusion hold if we take $\mathscr{A}^{\prime}$ to be the set of relaxed controls and we restrict ourselves to control problems (i.e. $B$ a singleton). Capuzzo Dolcetta-Ishii [15] and Loreti [40] used the theory of viscosity solutions in the same way to prove results of this kind for infinite horizon control problems. The argument does not hold in general for differential games because the problem with relaxed controls may have a different Hamiltonian (see e.g. [20]).

The two following applications are restricted to game theory. The definition of $V$ is not symmetric for the two players and indeed it describes a game where the first player has a slight advantage over the other one, so that $V$ is called the lower value of the game. We call $\Gamma$ the set of admissible (i.e. nonanticipating) strategies for the second player and define the upper value of the game:

$$
\widetilde{V}(x):=\sup _{\beta \in \Gamma} \inf _{a \in \mathscr{A}} P(x, a, \beta[a]) ;
$$

$\widetilde{R}$ is the corresponding upper capturability set.

A classical problem in the theory of differential games is the "existence of value", namely the coincidence of $V$ and $\widetilde{V}$ under the following Isaac's condition:

$$
\begin{array}{r}
H(x, p)=\widetilde{H}(x, p):=\max _{a \in A} \min _{b \in B}\{-f(x, a, b) \cdot p-h(x, a, b)\} \\
\text { for all } x, p \in \mathbf{R}^{N} .
\end{array}
$$


Results of this type have been one of the main contributions of the theory of viscosity solutions to differential games: see Barron-Evans-Jensen [9], EvansSouganidis [23], and Souganidis [43]. For our problem we have the following result which generalizes Theorem 2 in [7].

Corollary 7.5. Assume (H1).

(i) Comparison of upper and lower value: if $V$ and $\widetilde{V}$ are continuous on $\partial \mathscr{T}$, then $\widetilde{R} \subset \mathscr{R}$ and $V(x) \leq \widetilde{V}(x)$ for all $x \in \widetilde{R}$.

(ii) Existence of value: if (7.8) holds and $\widetilde{V}$ is continuous on $\partial \mathscr{T}$, then $\mathscr{K}=\widetilde{R}$ and $V=\widetilde{V}$.

Proof. (i) by the same arguments we mentioned in $\S 1, \widetilde{V}$ is a viscosity solution of

$$
\widetilde{H}(x, D V)=0 \quad \text { in } \widetilde{R} \backslash \mathscr{T} .
$$

The conclusion is then obtained by taking the Kruzkov transform of $V$ and $\widetilde{V}$, observing that $\mathscr{H} \geq \widetilde{\mathscr{H}}$ and applying Corollary 2.4 .

(ii) By the argument in the proof of Theorem 6.1, $\widetilde{V}$ is a continuous solution of (3.1) and therefore Theorem 3.1 provides the conclusion.

It is possible to show that if $g=0(\mathrm{H} 2)$ and $(\mathrm{H} 3)$ are sufficient conditions for the continuity of $\widetilde{V}$ as well.

Remark 7.6. Other definitions of value. It may be interesting to compare ElliottKalton's concept of value defined in $\S 1$ with any one among the many others known in the literature $[24,25,35,11]$. If another concept of the value $V^{\prime}$ satisfies the dynamic programming principle, then it is a viscosity solution of $H(x, D V)=0$ in $\mathscr{R}^{\prime} \backslash \mathscr{T}$. If $V^{\prime}$ is continuous on $\partial \mathscr{T}$ and goes to $+\infty$ on $\partial \mathscr{R}^{\prime}$, then our theory implies that $\mathscr{R}^{\prime}=\mathscr{R}$ and $V^{\prime}=V$.

Remark 7.7. Discontinuous value functions. It is easy to see that a lack of local controllability on some points of $\partial \mathscr{T}$ leads to value functions which do not tend to $+\infty$ on $\partial \mathscr{R}$ or which are discontinuous. The first event occurs for instance for the minimum time function in the classical Zermelo Navigation Problem reported by Caratheodory [16, $\S 458]$. The second event can be easily obtained by a simple modification of Zermelo's problem; another example can be found in Basar-Olsder [10, Chapter 5].

Clearly our global theory does not apply in these cases, but the local theory of $\S 4$ does apply near points of $\partial \mathscr{T}$ where there is some controllability. For instance Theorem 6.5 applies to both the examples above, and characterizes $T$ as the unique continuous solution of the Bellman equation in some neighborhood of the "good" part of $\partial \mathscr{T}$.

The only global uniqueness result for discontinuous solutions we know of at the present time is obtained by combining the Kruzkov transform with a theorem of Barles-Perthame [8], and it holds for the minimum time problem with smooth target. Its extension to games and to more general control problems seems out of reach at the moment. 
Remark 7.8. Approximation problems. Some ideas of this paper have already been used in [5] for proving the convergence of a discrete approximation to the minimum time function. The local theory in $\S 4$ can be used to approximate any given problem by one having a bounded invariant set, which is a necessary condition for implementing the approximation algorithm on a computer (see [6]). More general results involving differential games will be tackled in the future. We recall that the methods of comparison theorems for viscosity solutions can also be used for estimating the rate of convergence of various approximations (see e.g. Capuzzo-Dolcetta-Ishii [15]).

Remark 7.9. On the free boundary $\partial \mathscr{R}$. In general the free boundary $\partial \mathscr{R}$ does not have any regularity. In fact for any given $\Omega \supset \mathscr{T}, \mathscr{T}$ closed, $\Omega$ open and connected, we now show an example of controlled system $f(x, a)$ such that $\mathscr{R}=\Omega$. It suffices to define $d(x)=\operatorname{dist}\left(x, \Omega^{c}\right)$ and take

$$
f(x, a)=d(x) a, \quad a \in B(0,1) \subset \mathbf{R}^{N} .
$$

We can also choose a globally bounded dynamics by setting $f(x, a)=$ $(d(x) \wedge 1) a$.

In the theory of free boundary problems it is often possible to derive some additional conditions which the solution has to satisfy on the free boundary. Here we can do this for the transform of the capture time, $v=1-\exp (-T)$, which satisfies the Dirichlet boundary condition $v=1$ on $\partial \mathscr{R}$ and

$$
\min _{b \in B} \max _{a \in A}\{-f(x, a, b) \cdot D v\}=0 \text { on } \partial \mathscr{R},
$$

in the viscosity sense. Similar boundary conditions have been introduced by Soner [42] for control problems with state-constraints (see also [40] and the references therein).

\section{REFERENCES}

1. A. Bacciotti, Sulla continuità della funzione tempo minimo, Boll. Un. Mat. Ital. B 15 (1978), 859-868.

2. $\ldots$, Linear systems with piecewise constant controls, Boll. Un. Mat. Ital. A 18 (1981), 102-105.

3. __ Fondamenti geometrici della teoria della controllabilità, Quad. Unione Mat. Italiana, vol. 31, Pitagora, Bologna, 1985.

4. M. Bardi, $A$ boundary value problem for the minimum time function, SIAM J. Control 27 (1989), 776-785.

5. M. Bardi and M. Falcone, An approximation scheme for the minimum time function, SIAM J. Control 28 (1990), 950-965.

6. _ (in preparation).

7. M. Bardi and P. Soravia, A PDE framework for differential games of pursuit-evasion type, in Differential Games and Applications (T. Basar and P. Bernhard, eds.), Lecture Notes in Control and Inform. Sci. 144, Springer-Verlag, 1990, pp. 62-71.

8. G. Barles and B. Perthame, Discontinuous solutions of deterministic optimal stopping time problems, RAIRO Modél. Math. Anal. Numér. 21 (1987), 557-579. 
9. E. N. Barron, L. C. Evans and R. Jensen, Viscosity solutions of Isaacs' equations and differential games with Lipschitz controls, J. Differential Equations 53 (1984), 213-233.

10. T. Basar and G. J. Olsder, Dynamic non-cooperative game theory, Academic Press, New York, 1982.

11. L. D. Berkovitz, Differential games of generalized pursuit and evasion, SIAM J. Control 24 (1986), 361-373.

12. $\_$, Characterization of the values of differential games, Appl. Math. Optim. 17 (1988), 177-183.

13. A. Bressan, Sulla funzione tempo minimo nei sistemi nonlineari, Atti Accad. Naz. Lincei Mem. Cl. Sci. Fis. Mat. Natur. Sez. Ia 66 (1979), 383-388.

14. P. Cannarsa and H. M. Soner, On the singularities of viscosity solutions to Hamilton-JacobiBellmann equations, Indiana Univ. Math. J. 36 (1987), 501-524.

15. I. Capuzzo Dolcetta and H. Ishii, Approximate solutions of the Bellman equation of deterministic control theory, Appl. Math. Optim. 11 (1984), 161-181.

16. C. Caratheodory, Calculus of variations and partial differential equations of the first order, 2nd english ed., Chelsea, New York, 1982.

17. M. C. Crandall, L. C. Evans, and P. L. Lions, Some properties of viscosity solutions of Hamilton-Jacobi equations, Trans. Amer. Math. Soc. 282 (1984), 487-502.

18. M. C. Crandall, H. Ishii and P. L. Lions, Uniqueness of viscosity solutions revisited, J. Math. Soc. Japan 39 (1987), 581-596.

19. M. C. Crandall and P. L. Lions, Viscosity solutions of Hamilton-Jacobi equations, Trans. Amer. Math. Soc. 277 (1983), 1-42.

20. R. J. Elliott and N. J. Kalton, The existence of value in differential games, Mem. Amer. Math. Soc. No. 126 (1972).

21. L. C. Evans and H. Ishii, Differential games and nonlinear first order PDE on bounded domains, Manuscripta Math. 49 (1984), 109-139.

22. L. C. Evans and M. R. James, The Hamilton-Jacobi-Bellman equation for time-optimal control, SIAM J. Control 27 (1989), 1477-1489.

23. L. C. Evans and P. E. Souganidis, Differential games and representation formulas for solutions of Hamilton-Jacobi equations, Indiana Univ. Math. J. 33 (1984), 773-797.

24. W. Fleming, The convergence problem for differential games, J. Math. Anal. Appl. 3 (1961), 102-116.

25. A. Friedman, Differential games, Wiley, New York, 1971.

26. E. Giusti, On the equation of surfaces of prescribed mean curvature, Invent. Math. 46 (1978), 111-137.

27. O. Hajek, Pursuit games, Academic Press, New York, 1975.

28. H. Hermes, Feedback synthesis and positive, local solutions to Hamilton-Jacobi-Bellman equations, Analysis and Control of Nonlinear Systems (C. I. Byrnes, C. F. Martin and R. E. Saecks, eds.), North-Holland, Amsterdam, 1988, pp. 155-164.

29. H. Hermes and J. P. LaSalle, Functional analysis and time optimal control, Academic Press, New York, 1969.

30. R. Isaacs, Differential games, Wiley, New York, 1965.

31. H. Ishii, Uniqueness of unbounded viscosity solutions of Hamilton-Jacobi equations, Indiana Univ. Math. J. 33 (1984), 721-748.

32. __ Perron's method for Hamilton-Jacobi equations, Duke Math. J. 55 (1987), 369-384.

33. _ Representation of solutions of Hamilton-Jacobi equations, Nonlinear Anal. T.M.A. 12 (1988), 121-146.

34. __ A boundary value problem of the Dirichlet type for Hamilton-Jacobi equations, Ann. Scuola Norm. Sup. Pisa Cl. Sci. (4) 16 (1989), 105-135.

35. N. Krassovski and A. Soubbotine, Jeux différentiels, Mir, Moscow, 1977. 
36. S. N. Kruzkov, Generalized solutions of the Hamilton-Jacobi equations of the eikonal type. I, Math. USSR-Sb. 27 (1975), 406-445.

37. J. P. LaSalle, The time-optimal control problem, in Theory of Non-Linear Oscillations, vol. 5, Princeton Univ. Press, Princeton, N.J., 1958, pp. 1-24.

38. P. L. Lions, Generalized solutions of Hamilton-Jacobi equations, Pitman, Boston, 1982.

39. P. L. Lions and P. E. Souganidis, Differential games, optimal control and directional derivatives of viscosity solutions of Bellman's and Isaacs' equations, SIAM J. Control 23 (1985), 566-583.

40. P. Loreti, Some properties of constrained viscosity solutions of Hamilton-Jacobi equations, SIAM J. Control 25 (1987), 1244-1252.

41. N. N. Petrov, Controllability of autonomous systems, Differential Equations 4 (1968), 311317.

42. H. M. Soner, Optimal control problems with state-space constraints, I \& II, SIAM J. Control 24 (1987), 551-561 and 1110-1122.

43. P. E. Souganidis, Max-min representations and product formulas for the viscosity solutions of Hamilton-Jacobi equations with applications to differential games, Nonlinear Anal. T.M.A. 9 (1985), 217-257.

44. N. N. Subbotina, A. I. Subbotin and V. E. Tret' jakov, Stochastic and deterministic control. Differential inequalities, Problems Control Inform. Theory 14 (1985), 405-419.

45. A. I. Subbotin and A. M. Tarasyev, Stability properties of the value function of a differential game and viscosity solutions of Hamilton-Jacobi equations, Problems Control Inform. Theory 15 (1986), 457-463.

46. H. Sussmann, A general theorem on local controllability, SIAM J. Control 25 (1987), 158194.

47. A. Bressan, On a bang-bang principle for nonlinear systems, Suppl. Boll. Un. Mat. Ital. 1 (1980), 53-59.

Dipartimento di Matematica Pura ed Applicata, Universita di Padova, via Belzoni 7 , I-35131 Padova, Italy 